November 20, 2018

\title{
Strong Shock Waves and Nonequilibrium Response in a One-dimensional Gas: a Boltzmann Equation Approach
}

\author{
Pablo I. Hurtado \\ Department of Physics, Boston University, Boston, MA 02215, USA, and \\ Institute Carlos I for Theoretical and Computational Physics, \\ Universidad de Granada, 18071 Granada, Spain.
}

\begin{abstract}
We investigate the nonequilibrium behavior of a one-dimensional binary fluid on the basis of Boltzmann equation, using an infinitely strong shock wave as probe. Density, velocity and temperature profiles are obtained as a function of the mixture mass ratio $\mu$. We show that temperature overshoots near the shock layer, and that heavy particles are denser, slower and cooler than light particles in the strong nonequilibrium region around the shock. The shock width $\omega(\mu)$, which characterizes the size of this region, decreases as $\omega(\mu) \sim \mu^{1 / 3}$ for $\mu \rightarrow 0$. In this limit, two very different length scales control the fluid structure, with heavy particles equilibrating much faster than light ones. Hydrodynamic fields relax exponentially toward equilibrium, $\phi(x) \sim \exp [-x / \lambda]$. The scale separation is also apparent here, with two typical scales, $\lambda_{1}$ and $\lambda_{2}$, such that $\lambda_{1} \sim \mu^{1 / 2}$ as $\mu \rightarrow 0$, while $\lambda_{2}$, which is the slow scale controlling the fluid's asymptotic relaxation, increases to a constant value in this limit. These results are discussed at the light of recent numerical studies on the nonequilibrium behavior of similar 1d binary fluids.
\end{abstract}

\section{INTRODUCTION}

When a piston moves at constant velocity into a fluid, it generates a shock wave. [1] This is a strong perturbation that drives the system far from equilibrium. Studying the structural properties of the shock wave, and how equilibrium is restored behind it, we may extract valuable information on the fluid's transport properties, the damping of nonequilibrium fluctuations, etc. That is, we may use the shock wave as a probe to better understand the nonequilibrium response of the fluid. This is particularly appealing for one-dimensional (1d) systems. Their nonequilibrium behavior has attracted much attention during the last years. [2]-[19] This follows from two main reasons. On one hand, the dimensional constrain characteristic of $1 \mathrm{~d}$ systems plays an essential role in many real structures, ranging from carbon nanotubes 44 to anisotropic crystals [5], magnetic systems 6] solid copolymers [7], semiconductor wires [8], zeolites 9], Bose-Einstein condensates 10], colloids in narrow channels 11], DNA, nanofluids, etc. 2] On the other hand, the simplicity and versatility of $1 \mathrm{~d}$ models allow one to tackle fundamental questions in nonequilibrium statistical mechanics, as those related to irreversibility, normal transport, equipartition, local thermodynamic equilibrium, etc. [2]

In this way, it has been found that the low dimensionality can give rise to anomalous transport properties in $1 \mathrm{~d}$ fluids. To be more specific, the single-file (SF) constraint characteristic of $1 \mathrm{~d}$ fluids introduces strong correlations between neighboring particles, which asymptotically suppress mass transport 11 and enhance heat conduction 12, 13, 14, 15, 16, 17, 18, 19. In particular, it is currently believed (see however 12,13 ) that $1 \mathrm{~d}$ fluids with momentum-conserving interactions and non-zero total pressure exhibit a thermal conductivity $\kappa$ that diverges as $\kappa \sim L^{\gamma}$ when the system size $L \rightarrow \infty$. 14, 15, 16, 17, 18] However, there is no complete agreement yet on the exponent $\gamma>0$ : there exist strong numerical and theoretical evidences pointing out that $\gamma=1 / 3$ [14, 15], although mode-coupling theories and Boltzmann equation analysis predict $\gamma=2 / 5$ [16, 17], and other numerical results are closer to $\gamma=1 / 4$ 18]. Conservation of momentum seems to be crucial; as soon as this symmetry is broken, normal heat transport is recovered. 19] Moreover, momentum-conserving $1 \mathrm{~d}$ fluids typically exhibit other anomalies when perturbed away from equilibrium, as for instance dynamic non-equipartition of energy, emergence of multiple relaxation scales, energy localization, etc. 12, 13, 15, 18, 20] In general, the behavior of 1d fluids far from equilibrium still poses many intriguing questions which require further investigation.

In this paper we probe the nonequilibrium response of a $1 \mathrm{~d}$ model gas. In particular, we address the problem of an infinitely strong shock wave propagating into a 1d binary fluid, within the context of Boltzmann equation. 21, 22, 23. The shock wave characterizes the transition between two different asymptotic equilibrium states of the fluid. For strong shock waves (to be specified latter), this transition happens within molecular scales, so kinetic (Boltzmann) equations must be used. In fact, one may think of the shock wave problem as the simplest case study dominated by the nonlinearity of Boltzmann equation. 26] As we will see below, Boltzmann equation provides a powerful tool to investigate the observed structural and relaxational anomalies in $1 \mathrm{~d}$ fluids. Kinetic theory methods have been applied with success to study strong shock waves in high-dimensional 
simple fluids. 24, 25, 26] However, in one dimension, a simple (i.e. mono-component) fluid constitutes a pathological limit. 27] This follows from the fact that particles with equal masses in $1 \mathrm{~d}$ interchange their velocities upon collision, so a 1d mono-component fluid can be regarded to a large extent as an ideal gas of non-interacting particles. 28. We may restore ergodicity and relaxation in velocity space by introducing different masses. The simplest case is that of a binary mixture. In particular, we study in this paper a binary 1d fluid composed by two species of hard-point particles, with masses $m_{1}<m_{2}$ and equal concentrations. Energy transport in this model has already been extensively studied via molecular dynamics simulations. 12, 13, 15, 18, 37.

The structure of the paper is as follows. In section II, we write down the relevant Boltzmann equation for our 1d binary fluid, and extend a method by H. Grad 24, 26 to study the structure of an infinitely strong shock wave propagating through the fluid. The results of this approach are described in section III. In particular, we study there the shock hydrodynamic profiles, paying special attention to the shock width and the relaxation toward equilibrium, as a function of the mass ratio. Finally, in section IV we discuss our results.

\section{THE SHOCK-WAVE PROBLEM}

The Boltzmann equation for a 1d binary fluid in a reference frame moving with the shock wave reads, 21, 22, $23,26,29$

$$
\begin{aligned}
& v \frac{\mathrm{d} f_{1}}{\mathrm{~d} x}=\mathcal{Q}_{1}\left(f_{1}, f_{2}\right)-f_{1} \nu\left(f_{2}\right), \\
& v \frac{\mathrm{d} f_{2}}{\mathrm{~d} x}=\mathcal{Q}_{2}\left(f_{2}, f_{1}\right)-f_{2} \nu\left(f_{1}\right) .
\end{aligned}
$$

Here $f_{i}(x, v)$ is the probability density for finding a particle of type $i=1,2$ (i.e. mass $m_{i}$ ) at position $x$ with velocity $v$, and $\mathcal{Q}_{i}$ and $\nu$ represent the gain term and collision frequency in the collision operator, respectively. They are defined as,

$$
\begin{aligned}
\mathcal{Q}_{i}\left(f_{i}, f_{j}\right) & =\int_{-\infty}^{+\infty} \mathrm{d} w|v-w| f_{i}\left(x, v_{i}^{\prime}\right) f_{j}\left(x, w_{i}^{\prime}\right), \\
\nu\left(f_{i}\right) & =\int_{-\infty}^{+\infty} \mathrm{d} w|v-w| f_{i}(x, w) .
\end{aligned}
$$

Index $j$ refers here to particle species other than $i$, and $\left(v_{i}^{\prime}, w_{i}^{\prime}\right)$ are pre-collisional velocities that after collision give rise to velocities $(v, w)$ for the pair $(i, j)$, namely,

$$
\begin{aligned}
v_{i}^{\prime}(v, w) & =\frac{\left(m_{i}-m_{j}\right) v+2 m_{j} w}{m_{i}+m_{j}}, \\
w_{i}^{\prime}(v, w) & =\frac{\left(m_{j}-m_{i}\right) w+2 m_{i} v}{m_{i}+m_{j}} .
\end{aligned}
$$

Notice that eqs. (12)-(2) only include the effect of crosscollisions between the different species, and no selfcollisions between like particles. This is reflecting the one-dimensional character of our model fluid, and it is a main difference with Boltzmann equation for mixtures in higher dimensions.

The structure of the shock wave can be deduced from eqs. (11)-(2) subject to the boundary conditions,

$$
f_{i}(x, v) \rightarrow G_{i, \pm}(v) \quad \text { as } \quad x \rightarrow \pm \infty
$$

where

$$
G_{i, \pm}(v) \equiv n_{ \pm} \sqrt{\frac{m_{i}}{2 \pi T_{ \pm}}} \exp \left[-\frac{m_{i}\left(v-u_{ \pm}\right)^{2}}{2 T_{ \pm}}\right] .
$$

Here $n_{ \pm}, T_{ \pm}$and $u_{ \pm}$are respectively the number density, temperature and macroscopic velocity at $x \rightarrow \pm \infty$. 30. One usually chooses $u_{+}>0$, which implies (see below) $u_{-}>0$. This amounts to represent a flow of the fluid mixture from $-\infty$ (upstream or pre-shock region) to $+\infty$ (downstream or after-shock region).

We are particularly interested in the structure of an infinitely strong shock wave. In this way we can probe the response of the $1 \mathrm{~d}$ binary mixture in the strong nonequilibrium regime, far from the linear response region. 22 The strength of a shock may be defined in several ways. One of them is based on the ratio of downstream to upstream pressures, $r=p_{+} / p_{-}$, where $p_{ \pm}=n_{ \pm} T_{ \pm}$. Weak shocks have a ratio $r$ close to unity. In this case, the shock thickness is large as compared to the mean free path, and continuum approximations of the type of Navier-Stokes (or Euler, Burnett, etc.) may be used to characterize the shock structure. 1] However, as $r$ grows the shock thickness becomes comparable to the mean free path, making inappropriate the application of hydrodynamic approximations. In this case kinetic equations, as those in (1)(2), must be used instead. We pay attention in what follows to the limit $r \rightarrow \infty$, or equivalently $T_{-} \rightarrow 0$.

The parameters entering the two limiting Maxwellians (7) are related due to conservation of particle number, total momentum and total energy. Such conservation laws can be expressed as the constancy of the corresponding fluxes, which yields,

$$
\begin{aligned}
n_{+} u_{+} & =n_{-} u_{-}, \\
n_{+}\left[\left(m_{1}+m_{2}\right) u_{+}^{2}+2 T_{+}\right] & =n_{-}\left[\left(m_{1}+m_{2}\right) u_{-}^{2}\right], \\
n_{+}\left[\left(m_{1}+m_{2}\right) u_{+}^{3}+6 u_{+} T_{+}\right] & =n_{-}\left[\left(m_{1}+m_{2}\right) u_{-}^{3}\right] .
\end{aligned}
$$

These are the Rankine-Hugoniot conditions, which relate the downstream and upstream values of the flow fields in the mixture. [1] Solving this system for the downstream asymptotic flow fields one finds, 31]

$$
n_{+}=2 n_{-}, \quad u_{+}=\frac{u_{-}}{2}, \quad T_{+}=\frac{1}{8}\left(m_{1}+m_{2}\right) u_{-}^{2} .
$$

Therefore, the downstream asymptotic density (velocity) is twice (half) the upstream one for $r \rightarrow \infty$. It must 
be noticed that these results come from the conservation laws characterizing the two-component fluid, and have nothing to do with the hydrodynamic behavior of the mixture. In this way it is not strange that even in nonhydrodynamic cases, as the equal mass version of our one-dimensional gas, 28 the results (9) hold.

The case of a infinitely strong shock has been studied in detail for mono-component gases in dimensions larger than one. 24, 26] Extending a successful hypothesis by H. Grad, 24] we suggest here that the problem of a shock wave in our $1 \mathrm{~d}$ mixture is in general well-defined in the limit $r \rightarrow \infty$, and that in this case the distribution functions $f_{i}$ can be decomposed into a singular part, $\hat{f}_{i}$, proportional to a Dirac $\delta$-function on the upstream velocity $u_{-}$, and a regular part, $\tilde{f}_{i}$, smoother than the former. This is because for $r \rightarrow \infty$, particles in the upstream region exhibit a well-defined fixed thermal velocity when measured on the downstream velocity scale. Hence,

$$
f_{i}=\hat{f}_{i}+\tilde{f}_{i}, \quad \text { with } \quad \hat{f}_{i}(x, v)=\hat{n}_{i}(x) \delta\left(v-u_{-}\right) .
$$

The singular particle densities $\hat{n}_{i}(x)$ control how important the singular component is at a given coordinate $x$. In particular, it is evident that $\hat{n}_{i}(x) \rightarrow n_{-}$as $x \rightarrow-\infty$, and $\hat{n}_{i}(x) \rightarrow 0$ as $x \rightarrow+\infty$. Introducing the above decomposition (10) into eqs. (11)-(2), we may split the original Boltzmann equations in two different sets, attending to the singular or regular character of the terms,

$$
\begin{aligned}
v \frac{\mathrm{d} \hat{f}_{i}}{\mathrm{~d} x}+\hat{f}_{i} \nu\left(\tilde{f}_{j}\right) & =\mathcal{Q}_{i}\left(\hat{f}_{i}, \hat{f}_{j}\right)-\hat{f}_{i} \nu\left(\hat{f}_{j}\right), \\
v \frac{\mathrm{d} \tilde{f}_{i}}{\mathrm{~d} x}-\mathcal{Q}_{i}\left(\tilde{f}_{i}, \hat{f}_{j}\right)- & \mathcal{Q}_{i}\left(\hat{f}_{i}, \tilde{f}_{j}\right)+\tilde{f}_{i} \nu\left(\hat{f}_{j}\right)= \\
& =\mathcal{Q}_{i}\left(\tilde{f}_{i}, \tilde{f}_{j}\right)-\tilde{f}_{i} \nu\left(\tilde{f}_{j}\right),
\end{aligned}
$$

with $i=1,2$. Eq. (12) for the regular component $\tilde{f}_{i}$ looks more complicated than the original Boltzmann equations (11) -(2). However, as advantage, it lacks the singular behavior characterizing for $r \rightarrow \infty$ the whole distribution $f_{i}$. This allows us to perform a simple local Maxwellian approximation for $\tilde{f}_{i}$ that yields meaningful results. In particular, we now assume,

$$
\tilde{f}_{i}(x, v)=\tilde{n}_{i}(x) \sqrt{\frac{m_{i}}{2 \pi \tilde{T}_{i}(x)}} \exp \left[-\frac{m_{i}(v-\tilde{u}(x))^{2}}{2 \tilde{T}_{i}(x)}\right] .
$$

Here $\tilde{n}_{i}(x), \tilde{u}(x)$ and $\tilde{T}_{i}(x)$ are the number density, velocity and temperature associated to $\tilde{f}_{i}$. Now $\tilde{n}_{i}(x) \rightarrow 0$ as $x \rightarrow-\infty$ and $\tilde{n}_{i}(x) \rightarrow n_{+}$as $x \rightarrow+\infty$. We have also assumed at this point that the regular flow velocity $\tilde{u}(x)$ does not depend on particle species. This assumption seems natural given the one-dimensional character of the system, which forces neighboring particles to move coherently on average. Therefore the problem of the shock wave structure reduces to computing the spatial dependence of 7 different flow fields, namely $\hat{n}_{i}(x), \tilde{n}_{i}(x), \tilde{T}_{i}(x)$ and $\tilde{u}(x), i=1,2$.

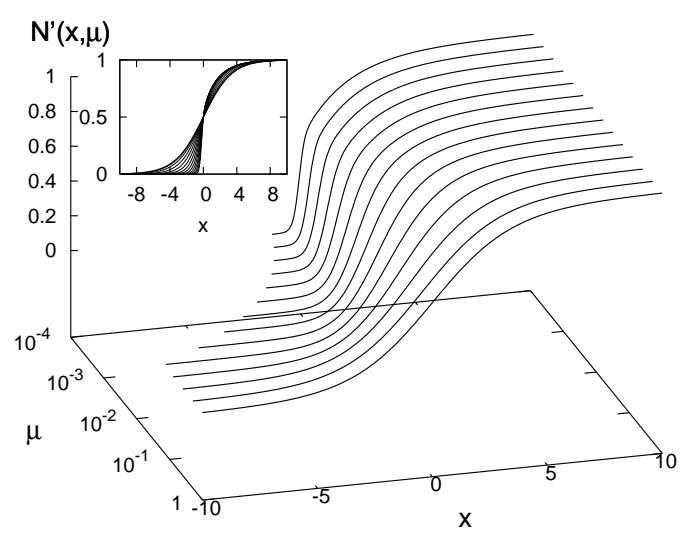

FIG. 1: Total density profiles (normalized) as a function of mass ratio $\mu$. Notice the logarithmic scale in the $\mu$-axis. The inset shows the same profiles in a $2 \mathrm{~d}$ plot for better comparison.

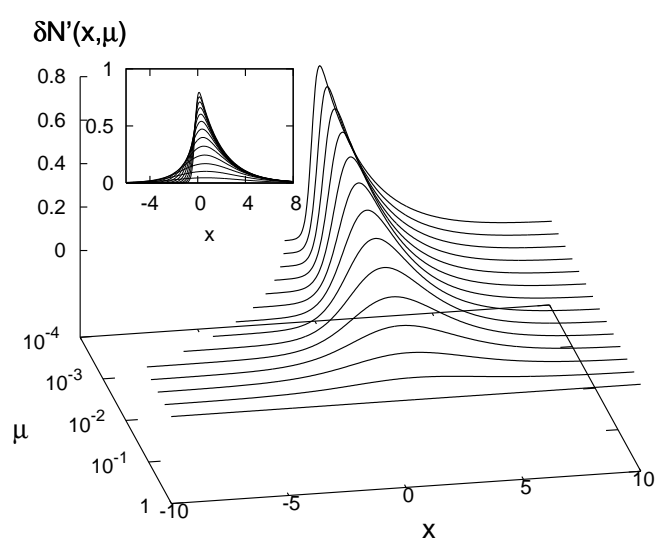

FIG. 2: Excess density profiles (normalized).

It is important to notice that the Maxwellian approximation (13) is not a local thermodynamic equilibrium (LTE) hypothesis, since it only affects the regular component $\tilde{f}_{i}(x, v)$ of the distribution function. The singular, delta-like component $\hat{f}_{i}(x, v)$ accounts for the most important nonequilibrium effects. However, it is natural to question the validity of (13) for the present strong nonequilibrium problem. This approximation is just the zeroth-order term in a formal expansion of $\tilde{f}_{i}(x, v)$ around local Maxwellian behavior, in the spirit of Sonine polynomials expansions, Gram-Charlier and Edgeworth series, etc. 32, 33, 34 One would expect that higher-order terms in this expansion could be relevant near the shock layer. In fact, such slight deviations have been observed in mono-component gases in higher dimensions. 26] However, these small corrections have negligible effects on the shock hydrodynamic profiles, [26] and therefore are not important for our discussion here.

We may use (10) and (13) to compute the local hy- 


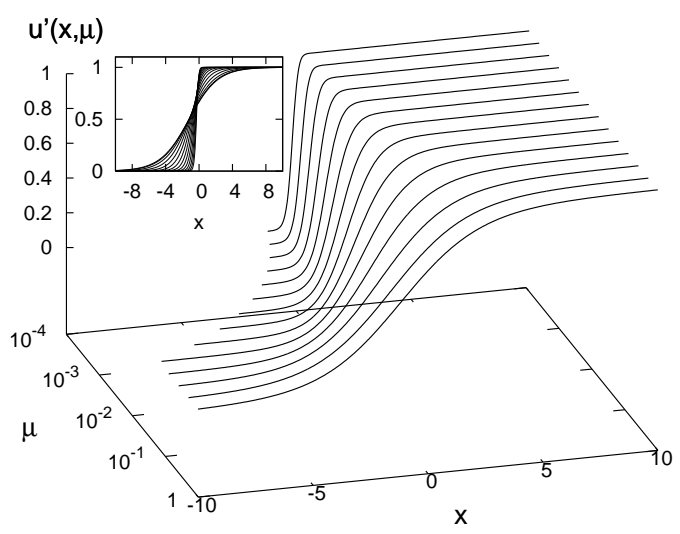

FIG. 3: Flow velocity profiles (normalized).

drodynamic currents. Let $J_{i, n}(x), J_{i, v}(x)$ and $J_{i, e}(x)$ be the particle, momentum and energy fluxes of species $i$ at position $x$, respectively. They can be written as,

$$
\begin{aligned}
J_{i, n}(x) & \equiv \int_{-\infty}^{+\infty} \mathrm{d} v v f_{i}(x, v)=\tilde{n}_{i} \tilde{u}+\hat{n}_{i} u_{-}, \\
J_{i, v}(x) & \equiv \int_{-\infty}^{+\infty} \mathrm{d} v m_{i} v^{2} f_{i}(x, v) \\
& =m_{i}\left(\tilde{n}_{i} \tilde{u}^{2}+\hat{n}_{i} u_{-}^{2}\right)+\tilde{n}_{i} \tilde{T}_{i} \\
J_{i, e}(x) & \equiv \int_{-\infty}^{+\infty} \mathrm{d} v m_{i} v^{3} f_{i}(x, v) \\
& =m_{i}\left(\tilde{n}_{i} \tilde{u}^{3}+\hat{n}_{i} u_{-}^{3}\right)+3 \tilde{u} \tilde{n}_{i} \tilde{T}_{i}
\end{aligned}
$$

(A trivial factor $\frac{1}{2}$ has been omitted in the definition of the energy flux for convenience). Given the constancy of the total fluxes along the system, we now write the Rankine-Hugoniot conditions as,

$$
\begin{array}{r}
\tilde{n}_{1} \tilde{u}+\hat{n}_{1} u_{-}=n_{-} u_{-}, \\
\tilde{n}_{2} \tilde{u}+\hat{n}_{2} u_{-}=n_{-} u_{-}, \\
m_{1}\left(\tilde{n}_{1} \tilde{u}^{2}+\hat{n}_{1} u_{-}^{2}\right)+m_{2}\left(\tilde{n}_{2} \tilde{u}^{2}+\hat{n}_{2} u_{-}^{2}\right)+ \\
+\tilde{p}_{1}+\tilde{p}_{2}=n_{-}\left(m_{1}+m_{2}\right) u_{-}^{2}, \\
m_{1}\left(\tilde{n}_{1} \tilde{u}^{3}+\hat{n}_{1} u_{-}^{3}\right)+m_{2}\left(\tilde{n}_{2} \tilde{u}^{3}+\hat{n}_{2} u_{-}^{3}\right)+ \\
+3 \tilde{u}\left(\tilde{p}_{1}+\tilde{p}_{2}\right)=n_{-}\left(m_{1}+m_{2}\right) u_{-}^{3},
\end{array}
$$

where $\tilde{p}_{i}=\tilde{n}_{i} \tilde{T}_{i}$ is the pressure of the regular component $\tilde{f}_{i}$. Defining $\tilde{M} \equiv m_{1} \tilde{n}_{1}+m_{2} \tilde{n}_{2}, \hat{M} \equiv m_{1} \hat{n}_{1}+m_{2} \hat{n}_{2}$, $M_{-} \equiv\left(m_{1}+m_{2}\right) n_{-}$, and $\tilde{P} \equiv \tilde{p}_{1}+\tilde{p}_{2}$, we obtain,

$$
\tilde{M} \tilde{u}=\hat{\Delta} u_{-}, \tilde{M} \tilde{u}^{2}+\tilde{P}=\hat{\Delta} u_{-}^{2}, \tilde{M} \tilde{u}^{3}+3 \tilde{u} \tilde{P}=\hat{\Delta} u_{-}^{3},
$$

where $\hat{\Delta} \equiv M_{-}-\hat{M}$. Using the first relation into the second equation one has $\tilde{P}=\hat{\Delta} u_{-}\left(u_{-}-\tilde{u}\right)$, and inserting this expression into the third equation yields (after division by $\left.\hat{\Delta} u_{-}\right) 2 \tilde{u}-3 u_{-} \tilde{u}+u_{-}^{2}=0$. This equation has solution $\tilde{u}=u_{-} / 2$ (the second root, $\tilde{u}=u_{-}$, is trivial and can be neglected as incompatible with the boundary

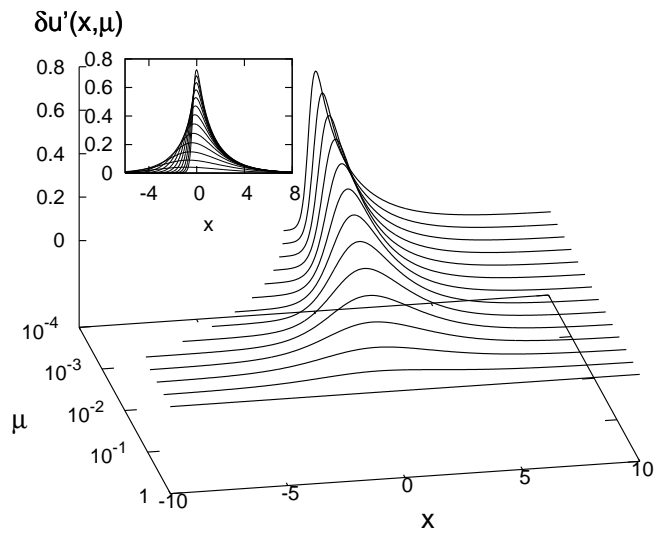

FIG. 4: Excess flow velocity profiles (normalized).

condition at $+\infty)$. Therefore the regular flow velocity is constant along the system, and equal to its asymptotic value at $+\infty, u_{+}$. Using this in eqs. (17) (18) one has,

$$
\begin{aligned}
\tilde{n}_{i}(x) & =2\left[n_{-}-\hat{n}_{i}(x)\right], \quad i=1,2 \\
\tilde{u}(x) & =\frac{1}{2} u_{-} .
\end{aligned}
$$

Combination of these results with $\tilde{P}=\hat{\Delta} u_{-}\left(u_{-}-\tilde{u}\right)$ yields a relation for the regular pressures, namely,

$$
\tilde{p}_{1}+\tilde{p}_{2}=\frac{u_{-}^{2}}{4}\left(m_{1} \tilde{n}_{1}+m_{2} \tilde{n}_{2}\right) .
$$

These manipulations are intended to express all remaining flow fields in terms of the singular particle densities, $\hat{n}_{i}(x)$. Equations for these singular densities can be derived integrating with respect to $v$ eq. (11) for the singular component $\hat{f}_{i} \cdot 35$ This yields,

$$
u_{-} \frac{\mathrm{d} \hat{n}_{i}(x)}{\mathrm{d} x}=2 \alpha_{j}(x) \hat{n}_{i}(x)\left[\hat{n}_{j}(x)-n_{-}\right],
$$

where,

$\alpha_{i}(x) \equiv \frac{u_{-}}{2} \operatorname{erf}\left[\sqrt{\frac{m_{i}}{2 \tilde{T}_{i}(x)}} \frac{u_{-}}{2}\right]+\sqrt{\frac{2 \tilde{T}_{i}(x)}{\pi m_{i}}} \exp \left[-\frac{m_{i} u_{-}^{2}}{8 \tilde{T}_{i}(x)}\right](26)$

and where we have used results (22) and (23). To completely specify the flow fields, we need another expression relating the regular pressures $\tilde{p}_{i}$ to the other fields. This relation can be derived studying the momentum and energy transfer from species $i$ to species $j$. Multiplying eq. (1) by $m_{i} v$ (momentum transfer) or $m_{i} v^{2}$ (energy transfer) and integrating over $v$, we arrive to differential equations for $J_{i, v}(x)$ and $J_{i, e}(x)$ of the form,

$$
\begin{aligned}
\frac{\mathrm{d} J_{i, v}}{\mathrm{~d} x} & =\Pi_{i}(x), \\
\frac{\mathrm{d} J_{i, e}}{\mathrm{~d} x} & =\Xi_{i}(x),
\end{aligned}
$$


where the fluxes are defined in eqs. (15)-(16) and,

$$
\begin{aligned}
\Pi_{i}(x) & \equiv \int_{-\infty}^{+\infty} \mathrm{d} v m_{i} v\left[\mathcal{Q}_{i}\left(f_{i}, f_{j}\right)-f_{i} \nu\left(f_{j}\right)\right], \\
\Xi_{i}(x) & \equiv \int_{-\infty}^{+\infty} \mathrm{d} v m_{i} v^{2}\left[\mathcal{Q}_{i}\left(f_{i}, f_{j}\right)-f_{i} \nu\left(f_{j}\right)\right] .
\end{aligned}
$$

Since the total momentum flux, $J_{1, v}(x)+J_{2, v}(x)$, and total energy flux, $J_{1, e}(x)+J_{2, e}(x)$, are constants along the line (Rankine-Hugoniot conditions), the above differential equations, (27)-(28), express the transfer of momentum and energy from species $i$ to species $j$ at a given point $x$. Using the expressions for $J_{i, v}(x)$ and $J_{i, e}(x)$ in (15) - (16), it is easy to show that $3 \tilde{u} J_{i, v}(x)-J_{i, e}(x)=$ $\frac{1}{2} m_{i} n_{-} u_{-}^{3}=$ constant, and therefore,

$$
3 \tilde{u} \Pi_{i}(x)-\Xi_{i}(x)=0 .
$$

This last equation will give us the desired extra relation for the regular pressures. In general, the integrals in (29) - (30) cannot be performed due to the velocitydependent collision kernel $|v-w|$ appearing in the definition of $\mathcal{Q}_{i}$ and $\nu$, see (3) and (4). In order to continue our derivation, we now approximate this kernel by a generic velocity-independent kernel $\sigma(x)$, much in the spirit of Maxwell molecules. 29]

Using this assumption we can solve the above integrals, obtaining,

$$
\begin{aligned}
\Pi_{i}(x) & =\frac{2 m_{i} m_{j}}{m_{i}+m_{j}} n_{-} u_{-} \sigma(x)\left(N_{i}-N_{j}\right), \\
\Xi_{i}(x) & =\frac{m_{i} m_{j}}{\left(m_{i}+m_{j}\right)^{2}} \sigma(x)\left\{u _ { - } ^ { 2 } \left[\left(m_{i}+2 m_{j}\right) \tilde{n}_{i} \hat{n}_{j}\right.\right. \\
& \left.\left.-\left(m_{j}+2 m_{i}\right) \hat{n}_{i} \tilde{n}_{j}\right]-4\left[N_{j} \tilde{p}_{i}-N_{i} \tilde{p}_{j}\right]\right\},
\end{aligned}
$$

where we have defined the species total number density, $N_{i}(x) \equiv \tilde{n}_{i}(x)+\hat{n}_{i}(x)=2 n_{-}-\hat{n}_{i}(x), i=1,2$. Within this approximation, eq. (31) reduces to,

$$
N_{j} \tilde{p}_{i}-N_{i} \tilde{p}_{j}=\frac{u_{-}}{8}\left(m_{j}-m_{i}\right)\left(\tilde{n}_{i} \hat{n}_{j}+\hat{n}_{i} \tilde{n}_{j}\right),
$$

which is the second relation between the regular pressures we were looking for. Together with (24), this yields,

$$
\tilde{p}_{i}=\frac{u_{-}^{2}\left[\left(m_{j}-m_{i}\right)\left(\tilde{n}_{i} \hat{n}_{j}+\hat{n}_{i} \tilde{n}_{j}\right)+2 \tilde{M} N_{i}\right]}{8\left(N_{i}+N_{j}\right)},
$$

where we have used the previous definition $\tilde{M} \equiv m_{i} \tilde{n}_{i}+$ $m_{j} \tilde{n}_{j}$. This result, based on the Maxwell velocity kernel approximation, gives predictions for the regular pressures which are very close $\forall x$ to the results obtained from the solution of eq. (31) based on the numerical evaluation of integrals (29)-(30), thus validating our approximation. The reason behind this good agreement is that Eq. (34) does not depend on the collision kernel $\sigma(x)$ chosen for the Maxwell-like approximation: the same equation is

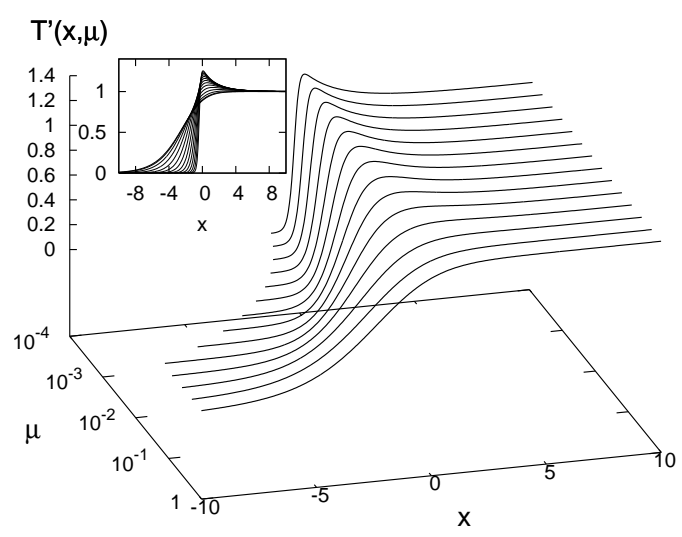

FIG. 5: Temperature profiles (normalized).

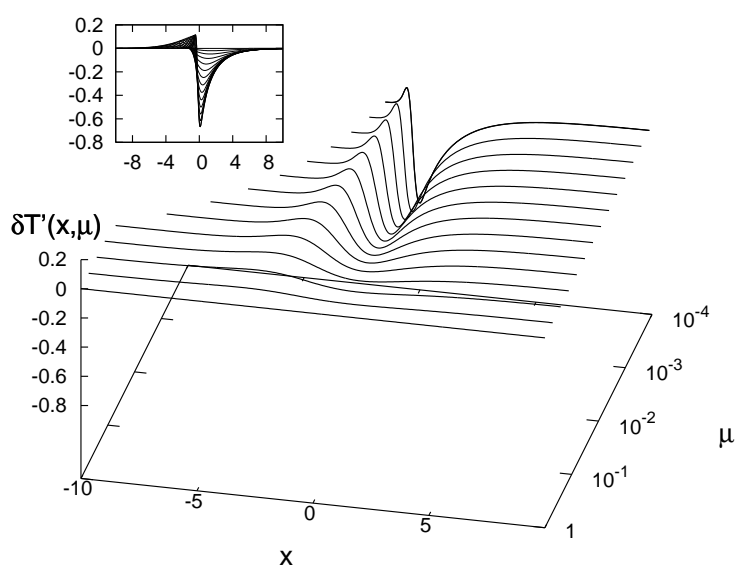

FIG. 6: Excess temperature profiles (normalized).

found for any kernel $\sigma(x)$ one may think of, and in particular for the (unknown) optimal kernel which better approximates the real velocity-dependent kernel.

Recalling that $\tilde{n}_{i}=2\left(n_{-}-\hat{n}_{i}\right)$ and $\tilde{u}=\frac{1}{2} u_{-}$, eqs. (35) express the regular pressures $\tilde{p}_{i}=\tilde{n}_{i} \tilde{T}_{i}$, and hence the regular temperatures, in terms of the singular densities $\hat{n}_{i}$. Therefore the problem of the shock wave reduces to solving the coupled, strongly non-linear differential equations (25) for $\hat{n}_{i}(x), i=1,2$, since all other non-constant flow fields, $\tilde{n}_{i}$ and $\tilde{T}_{i}$, with $i=1,2$, can be written in terms of $\hat{n}_{i}$.

\section{RESULTS}

The strong non-linearities present in eqs. 251) prevent any full analytical solution to the shock wave problem, so numerical integration must be used instead. This procedure yields the singular densities $\hat{n}_{i}(x)$, from which all hydrodynamic flow fields follow. We are particularly interested here in the total number density $N(x)$, flow velocity $u(x)$, and temperature $T(x)$, and their par- 
ticularization to each species $\left(N_{i}(x), u_{i}(x)\right.$ and $T_{i}(x)$, $i=1,2$, respectively). The total number density is defined as $N(x) \equiv N_{1}(x)+N_{2}(x)$, where $N_{i}(x)=$ $\tilde{n}_{i}(x)+\hat{n}_{i}(x)$ has been defined above. The total flow velocity and temperature fields are defined respectively as $u(x) \equiv\left[\sum_{i} m_{i} N_{i}(x)\right]^{-1} \sum_{i} m_{i} N_{i}(x) u_{i}(x)$ and $T(x) \equiv$ $N(x)^{-1} \sum_{i} N_{i}(x) T_{i}(x),[22]$ where,

$$
\begin{aligned}
u_{i} & \equiv N_{i}^{-1} \int_{-\infty}^{+\infty} \mathrm{d} v v f_{i}(x, v)=\frac{n_{-} u_{-}}{N_{i}}, \\
T_{i} & \equiv N_{i}^{-1} \int_{-\infty}^{+\infty} \mathrm{d} v m_{i}[v-u(x)]^{2} f_{i}(x, v) \\
& =\frac{\tilde{n}_{i} \tilde{T}_{i}+m_{i}\left[\tilde{n}_{i}\left(\frac{1}{2} u_{-}-u\right)^{2}+\hat{n}_{i}\left(u_{-}-u\right)^{2}\right]}{N_{i}} .
\end{aligned}
$$

For a generic hydrodynamic flow field $\phi(x)$, we now define an associated normalized field as $\phi^{\prime}(x)=(\phi(x)-$ $\left.\phi_{-}\right) /\left(\phi_{+}-\phi_{-}\right)$, where $\phi_{ \pm}=\phi(x \rightarrow \pm \infty)$. This allows us to compare shock profiles for different mass ratios $\mu \equiv$ $m_{1} / m_{2} \in(0,1)$. In addition, we define the normalized excess flow fields as $\delta \phi^{\prime}(x) \equiv \phi_{2}^{\prime}(x)-\phi_{1}^{\prime}(x)$ to study the species dependence of the profiles. Our results for $N^{\prime}(x), u^{\prime}(x)$ and $T^{\prime}(x)$ are given in Figs. 1, 3 and 5, respectively, while the excess fields $\delta N^{\prime}(x), \delta u^{\prime}(x)$ and $\delta T^{\prime}(x)$ are depicted in Figs. 2, 4 and 6. It should be noted that we set the coordinate origin $x=0$ so that $N^{\prime}(x=0)=\frac{1}{2}$.

A general observation is that all the profiles get steeper, and the excess fields get more localized and peaked around $x=0$, as $\mu$ decreases. However, there are fundamental differences for the different hydrodynamic quantities. The density flow field, $N^{\prime}(x)$, converges toward a limiting shape as $\mu \rightarrow 0$, characterized by a sudden increase of density from $N^{\prime}\left(x \rightarrow 0_{-}\right)=0$ to $N^{\prime}\left(x \rightarrow 0_{+}\right)=\frac{1}{2}$, followed by a much slower relaxation toward the asymptotic value $N^{\prime}(x \rightarrow+\infty)=1$, see Fig. 1. On the other hand, the flow velocity profile $u(x)$ converges toward a step-like function localized at $x=0$, Fig. 3, while the temperature field, Fig. 5, exhibits an overshoot which sharpens and increases as $\mu \rightarrow 0$. In addition, all profiles are markedly asymmetrical. Fig. 7 exhibits all fields for several mass ratios.

The excess profiles contain the information about the relative local distribution of the hydrodynamic fields between the species. In particular, $\delta N^{\prime}(x)$ shows that the number density of heavy particles (type 2) around the shock layer is larger than that of light particles (type 1), see Fig. 2. This excess concentration of heavy particles around the shock is asymmetric, with most excess heavy particles lagging behind the shock, and it increases as $\mu \rightarrow 0$. On the other hand, the flow velocity $u_{1}(x)$ of light particles around the shock is larger than the velocity of heavy particles, see Fig. 4, the difference increasing with decreasing $\mu$ (notice here that $\delta u^{\prime}(x)>0$ involves $\left.u_{1}(x)>u_{2}(x)\right)$. Finally, the excess temperature field exhibits an interesting behavior, see Figs. 6
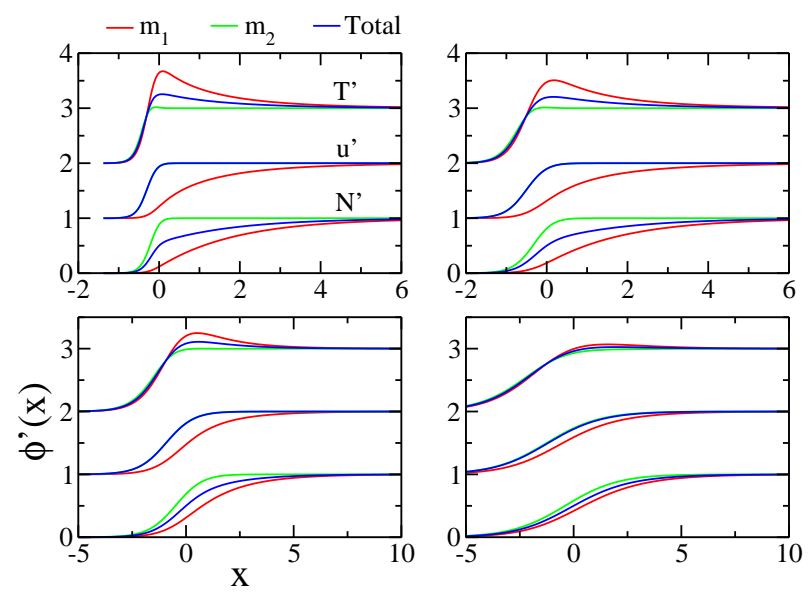

FIG. 7: (Color online) Density, velocity and temperature profiles for each species and the mixture. From left to right and top to bottom, $\mu=0.000125,0.001,0.016,0.128$. Velocity (temperature) profiles have been shifted one (two) units in the vertical axis for visual convenience.

and 7. First, $\delta T^{\prime}(x)$ is mostly negative near the shock, so heavy particles are cooler than light particles around the shock, despite the former are denser than the latter around $x=0$. More in detail, $\delta T^{\prime}(x)$ exhibits an oscillation around $x=0$, meaning that heavy particles are much colder than light particles behind the shock layer (the cooler the smaller $\mu$ is), while the situation is reversed in front of the shock, where heavy particles are now more energetic than light ones, see Fig. 6.

To better understand some of these observations, we can analyze the asymptotic behavior of eqs. 25). First, we study how the downstream binary fluid goes toward equilibrium by letting $x \gg 0$. In this case, the singular densities are very small, $\hat{n}_{i}(x \gg 0) \ll 1$, so we can linearize eqs. (25), obtaining a simple decoupled system of differential equations,

$$
u_{-} \frac{\mathrm{d} \hat{n}_{i}(x)}{\mathrm{d} x}=-2 \alpha_{j}(+\infty) n_{-} \hat{n}_{i}(x),
$$

with $i=1,2$, and where,

$$
\begin{aligned}
\alpha_{i}(+\infty) & =\frac{u_{-}}{2}\left[\operatorname{erf}\left(\sqrt{\frac{m_{i}}{m_{1}+m_{2}}}\right)\right. \\
& \left.+\sqrt{\frac{m_{1}+m_{2}}{\pi m_{i}}} \exp \left(-\frac{m_{i}}{m_{1}+m_{2}}\right)\right]
\end{aligned}
$$

is the limit of the amplitudes (26) as $x \rightarrow+\infty$. Therefore, we find $\hat{n}_{i}(x) \sim \exp \left[-x / \lambda_{j}\right]$ in this limit, with $\lambda_{j}=u_{-} /\left[2 n_{-} \alpha_{j}(+\infty)\right]$, so the downstream binary fluid reaches equilibrium exponentially fast, as expected from the uncorrelated nature of Boltzmann equation. 21, 22, 23. This equilibration process is characterized however by two different typical scales: $\lambda_{1}$ controls how heavy particles (type 2) relax asymptotically, while $\lambda_{2}$ controls the behavior of light particles (type 1 ). The $\mu$-dependence 


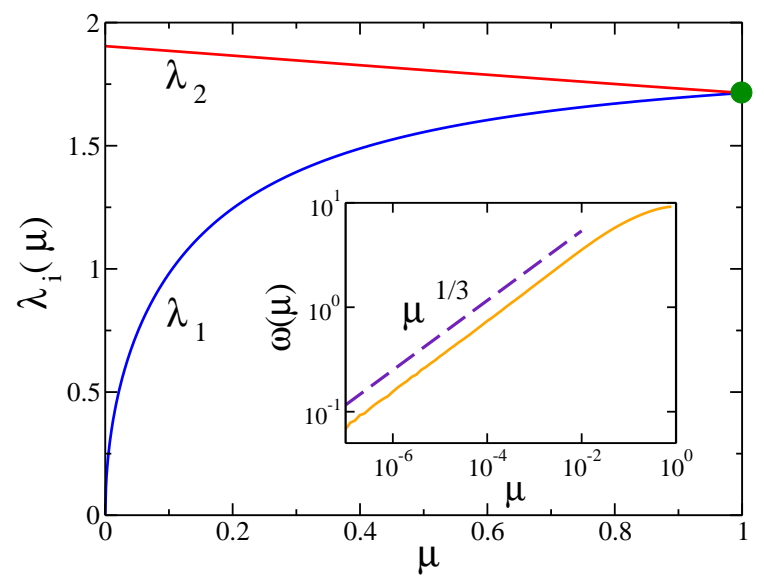

FIG. 8: (Color online) Main: Mass ratio dependence of the typical scales $\lambda_{i}$ controlling the exponential relaxation of the downstream binary fluid toward equilibrium. Notice that $\lambda_{1}$ scales as $\mu^{1 / 2}$ for small $\mu$. The solid dot signals the singular limit $\mu=1$, for which both typical scales formally diverge. Inset: Mass ratio dependence of the shock width $\omega$ in $\log -\log$ scale. The width decreases as $\omega \sim \mu^{1 / 3}$ for $\mu \rightarrow 0$.

of both typical scales is depicted in Fig. 8. Here we see that $\lambda_{2}>\lambda_{1} \forall \mu \in(0,1)$. In fact, $\lambda_{2}$ converges to a constant as $\mu \rightarrow 0$, while $\lambda_{1}$ goes asymptotically to 0 as $\mu^{1 / 2}$, see eq. (37). Therefore, a separation of scales emerges for very small mass ratios $\mu$, with both fast and slow evolution scales controlling the fluid relaxation. All the macroscopic hydrodynamic fields $\phi(x)$ relax asymptotically as $\exp \left[-x / \lambda_{2}\right]$, following the slowest relaxation scale associated to light particles, except for the number density for heavy particles, which relax much faster, as $\exp \left[-x / \lambda_{1}\right]$.

In the opposite limit, $\mu \rightarrow 1$, a singularity emerges. In fact, Boltzmann equation does not yield any useful information for $\mu=1$ in one dimension (1d): the gain and loss terms in the collision operator are equal in this case, so the collision term in Boltzmann equation is zero (see right hand side of Eqs. (1)-(2)). That is, collisions do not change the velocity distributions $f_{i}(x, v)$ in this limit, as expected from the fact that equal-mass particles exchange their velocities upon collision in 1d. Therefore there is no relaxation of hydrodynamic fields for $\mu=1$, and the relaxation scales $\lambda_{i}$ are not defined. Alternatively, this can be interpreted as a divergence of both $\lambda_{i}$. However, this is a singular behavior which emerges only for $\mu=1$. As soon as $\mu<1$, normal behavior is recovered. In particular, for $\mu$ slightly smaller than 1 , one expects both typical scales to be almost equal, as observed in Fig. 8.

The separation of scales described above for $\mu \rightarrow 0$ does not only emerge for $x \rightarrow+\infty$, but characterizes the profiles $\forall x$. In fact, the amplitudes $\alpha_{i}(x)$, see eqs. (26), are such that $\alpha_{1}(x) \gg \alpha_{2}(x) \forall x$ as $\mu \rightarrow 0$. Hence, in this limit the singular density $\hat{n}_{2}(x)$ changes at a much faster rate than $\hat{n}_{1}(x)$ does, see eqs. (25), so we can consider $\hat{n}_{1}(x)$ as a constant in the scale in which $\hat{n}_{2}(x)$ evolves. This effectively decouples again the differential equations (25) (though they are still highly non-linear), giving rise to a multi-scale relaxation process: (i) In a first step, heavy particles very quickly relax toward their equilibrium downstream asymptotic state while light particles remain close to the upstream state. (ii) This is followed by a relatively much slower relaxation of light particles toward equilibrium. A good illustration of this process can be found in the top-left panel in Fig. 7. This twoscales relaxation mechanism for $\mu \rightarrow 0$ explains the limiting shape described above for $N^{\prime}(x)$, see Fig. 1, as well as the the positive excess density $\delta N^{\prime}(x)$ in Fig. 2. In addition, it also explains the step-like limiting profile for the flow velocity: $u(x)$ is the weighted average of the species flow velocities $u_{i}(x)$, the weigh being proportional to the species mass, so for $\mu \rightarrow 0 u(x)$ coincides with $u_{2}(x)$, which is controlled by the fastest relaxation scale.

It seems also interesting to characterize the region where the fluid is far from equilibrium. A canonical measure of the scale or typical size associated to this region is given by the shock width $\omega$. The shock width is usually defined as the inverse of the maximum density profile derivative, $\omega(\mu) \equiv\left(\frac{\mathrm{d} N}{\mathrm{~d} x}\right)_{\max }^{-1}$. [1] It can be also defined as the standard deviation of the (peaked) distribution $A N^{\prime}(x)\left[1-N^{\prime}(x)\right]$, with $A$ a suitable normalization constant. Both definitions give equivalent results. The inset to Fig. 8 shows our results for $\omega(\mu)$. The shock width decreases as the mass ratio decreases, scaling as $\omega(\mu) \sim \mu^{1 / 3}$ for $\mu \rightarrow 0.36$ Therefore nonequilibrium effects are more localized around $x=0$ for smaller $\mu$. However, it is remarkable that despite the shock wave gets steeper ( $\omega$ decreases) as $\mu$ decreases, the typical scale characterizing relaxation toward equilibrium, $\lambda_{2}$, increases with $\mu$. Hence, although the strong nonequilibrium region is reduced as $\mu \rightarrow 0$, the consequent fluid evolution to equilibrium slows down.

\section{CONCLUSIONS}

In this paper we have studied the response of a onedimensional binary fluid to a strong shock wave propagating into it, on the basis of Boltzmann equation. This excitation drives the system far from equilibrium, therefore allowing us to investigate the structure of the fluid under nonequilibrium conditions and how equilibrium is regained asymptotically.

Extending to fluid mixtures an elegant method by $\mathrm{H}$. Grad, 24, 26] we have obtained the shock hydrodynamic profiles characterizing the transition between the two different asymptotic equilibrium states. In particular, we determine the flow fields as a function of the mixture mass ratio $\mu=m_{1} / m_{2} \in(0,1)$. We find in general that all profiles sharpen as $\mu \rightarrow 0$. The particle number den- 
sity field converges in this limit to an asymptotic shape characterized by a sudden step-like increase followed by a much slower relaxation to equilibrium. The flow velocity profile converges in turn to a step-like function, while the temperature field exhibits a characteristic overshoot which increases with decreasing $\mu$. In addition, the density (velocity) of heavy particles behind the shock is larger (smaller) than that of light particles, the differences increasing as $\mu \rightarrow 0$. On the other hand, heavy particles are much cooler than light particles right behind the shock, while the reverse situation holds in front of the shock, where heavy particles are now slightly more energetic than light ones.

In order to understand these results, we have performed an asymptotic analysis of our equations. This reveals the emergence of two very different typical length scales controlling relaxation as $\mu \rightarrow 0$. In this limit, the fluid evolves toward equilibrium in twos steps: (i) First, heavy particles quickly relax toward their asymptotic downstream equilibrium state, while light particles remain close to the upstream state; (ii) at a much slower pace, light particles come to equilibrium. In this way, light particles ultimately control the fluid's global equilibration. Far behind the shock layer, the hydrodynamic fields relax exponentially. Here the multi-scale relaxation mechanism is also apparent, with two typical exponential scales, $\lambda_{i}, i=1,2$. In particular, $\lambda_{1}$, associated to heavy particles, goes to zero as $\mu^{1 / 2}$, while $\lambda_{2}$, which controls the global relaxation to equilibrium, increases as $\mu \rightarrow 0$, converging to a constant. In addition, the size of the strong nonequilibrium region associated to the shock layer, as measured by the shock width $\omega(\mu)$, decreases as $\omega(\mu) \sim \mu^{1 / 3}$ for $\mu \rightarrow 0$. Therefore, the fluid's strong nonequilibrium behavior gets more localized around $x=0$ as $\mu$ decreases, despite its asymptotic relaxation to equilibrium, as given by $\lambda_{2}$, slows down with decreasing $\mu$.

These results are specially interesting at the light of recent numerical studies of similar 1d binary fluids. 12, 13, 15, 18, 20, 37] In particular, simulations show that whenever the 1d binary fluid is perturbed away from equilibrium, the consequent relaxation happens in such a way that light particles always tend to absorb more energy than heavy ones, as predicted here. In addition, light particles are observed to equilibrate more slowly, therefore controlling the system global relaxation. This supports the presence of two different relaxation scales for $\mu \rightarrow 0$, validating our theoretical results.

The success of our Boltzmann equation approach to describe some of the structural and relaxational anomalies observed in the $1 \mathrm{~d}$ diatomic fluid would suggest extending the present approach to understand its anomalous transport properties (see Section I) 12, 13, 14, 15, 16, 17, 18]. Unfortunately, this is not possible. Anomalous transport in $1 \mathrm{~d}$ is a direct consequence of the long-time powerlaw tails characterizing the decay of correlation func- tions in the fluid. The microscopic origin of these tails is associated to dynamically correlated sequences of collisions among fluid molecules, which are not described by Boltzmann equation. 38. That is, the main hypothesis on which Boltzmann equation is based, namely the molecular chaos hypothesis, 21, 22, 23] breaks correlations present in the fluid which are responsible of the power-law tails.

The strong correlations emerging in $1 \mathrm{~d}$ fluids, and its role in the shock wave problem, are interesting issue which deserve attention. 37, 39] Further work in this direction, taking into account these correlations in generalized kinetic theories, would be highly desirable to better understand the nonequilibrium behavior of simple onedimensional systems.

Acknowledgments: The author thanks P.L. Garrido and P.L. Krapivsky for useful comments and discussions, as well as the Spanish MEC for financial support.

[1] G.B. Whitham, Linear and Nonlinear Waves, WileyInterscience 1999.

[2] S. Lepri, R. Livi, A. Politi, Phys. Rev. Lett. 78, 18961899 (1997); ibid, Phys. Rep. 377, 1 (2003).

[3] V. Privman (ed.), Nonequilibrium Statistical Mechanics in One Dimension, Cambridge University Press, Cambridge (1997).

[4] J. Hone , M. Whitney, C. Piskoti, A. Zettl, Phys. Rev. B 59, R2514 (1999); P. Kim, L. Shi, A. Majumdar, P.L. McEuen, Phys. Rev. Lett. 87, 215502 (2001).

[5] D.T. Morelli, J. Heremans, M. Sakamoto, C. Uher, Phys. Rev. Lett. 57, 869 (1986); A. Smontara, J.C. Lasjaunias, R. Maynard, Phys. Rev. Lett. 77, 5397 (1996).

[6] A.V. Sologubenko, K. Giannò, H. R. Ott, A. Vietkine, A. Revcolevschi, Phys. Rev. B 64, 054412 (2001).

[7] H. Forsman, P. Anderson, J. Chem. Phys. 80, 2804 (1984).

[8] L.G.C. Rego, G. Kirczenow, Phys. Rev. Lett. 81, 232 (1998); D.M. Leitner, P.G. Wolynes, Phys. Rev. E 61, 2902 (2000).

[9] V. Kukla et al, Science 272, 702 (1996); K. Hahn, J. Kärger, V. Kukla, Phys. Rev. Lett. 76, 2762 (1996).

[10] M. Porter, Bull. Am. Phys. Soc. 50, 1047 (2005).

[11] T.E. Harris, J. Appl. Prob. 2, 323 (1965); D.G. Levitt, Phys. Rev. A 8, 3050 (1973); Q.-H. Wei, C. Bechinger, P. Leiderer, Science 287, 625 (2000); B. Cui, H. Diamant, B. Lin, Phys. Rev. Lett. 89, 188302 (2002); C. Lutz, M. Kollmann, C. Bechinger, Phys. Rev. Lett. 93, 026001 (2004).

[12] P.L. Garrido, P.I. Hurtado, B. Nadrowski, Phys. Rev. Lett. 86, 5486 (2001).

[13] P.L. Garrido, P.I. Hurtado, Phys. Rev. Lett. 88, 249402 (2002); A. Dhar, Phys. Rev. Lett. 88, 249401 (2002); P.L. Garrido, P.I. Hurtado, Phys. Rev. Lett. 89, 079402 (2002); H. Li, Y. Wang, H. Zhao, Phys. Rev. Lett. 89, 079401 (2002).

[14] O. Narayan, S. Ramaswamy, Phys. Rev. Lett. 89, 200601 (2002). 
[15] P. Grassberger, W. Nadler, L. Yang, Phys. Rev. Lett. 89, 180601 (2002); P. Cipriani, S. Denisov, A. Politi, Phys. Rev. Lett. 94, 244301 (2005)

[16] J.-S. Wang, B. Li, Phys. Rev. Lett. 92, 074302 (2004).

[17] A. Pereverzev, Phys. Rev. E 68, 056124 (2003).

[18] A. Dhar, Phys. Rev. Lett. 86, 3554 (2001); A.V.Savin, G.P. Tsironis, A.V. Zolotaryuk, Phys. Rev. Lett. 88, 154301 (2002); G. Casati, T. Prosen, Phys. Rev. E 67, 015203(R) (2003).

[19] B. Li, G. Casati, J. Wang, T. Prosen, Phys. Rev. Lett. 92, 254301 (2004).

[20] A. Kato, D. Jou, Phys. Rev. E 64, 052201 (2001).

[21] P. Résibois, M. de Leener, Classical Kinetic Theory of Fluids, John Wiley \& Sons, New York (1977).

[22] S.R. de Groot, P. Mazur, Nonequilibrium Thermodynamics, Dover, New York (1984).

[23] S. Harris, An Introduction to the Theory of the Boltzmann Equation, Dover, New York (2004).

[24] H. Grad, in Transport Theory, R. Bellman, G. Birkhoff and I. Abu-Shumays, eds., American Mathematical Society, Providence, R.I. (1969), pp. 269-308.

[25] H. Mott-Smith, Phys. Rev. 82, 885 (1951).

[26] C. Cercignani, A. Frezzotti and P. Grosfils, Phys. Fluids 11, 2757 (1999).

[27] D.W. Jepsen, J. Math. Phys. (N.Y.) 6, 405 (1965).

[28] For $m_{1}=m_{2}$, the fluid studied in this paper reduces to an ideal one-dimensional gas of non-interacting particles. This system is not ergodic, and lacks any relaxation mechanism in velocity space capable of driving the system toward local thermodynamic equilibrium.

[29] R. Dickman, J. Stat. Phys. 41, 607 (1985); O.J. Eder and M. Posch, Phys. Rev. A 34, 2283 (1986).

[30] Notice that the asymptotic number densities $n_{ \pm}$do not depend on the species, since we are interested here on a 1d binary fluid with equal concentrations of light and heavy particles.

[31] In this process we disregard a trivial solution $u_{+}=u_{-}$ which is incompatible with the boundary condition at $+\infty$.

[32] M. Abramowitz and I.A. Stegun, (eds.), Handbook of Mathematical Functions with Formulas, Graphs, and Mathematical Tables, 9th printing, Dover, New York (1972).

[33] S. Blinnikov and R. Moessner, Astron. \& Astrophys. Sup. 130, 193 (1998).

[34] See, for instance, E.W. Weisstein, Edgeworth series, from MathWorld-A Wolfram Web Resource, http://mathworld.wolfram.com/EdgeworthSeries.html

[35] Computing the time evolution of any moment of $f_{i}$ from eq. (11) gives rise always to the same equation for $\hat{n}_{i}(x)$, namely eq. (25).

[36] It should be noticed that, due to the separation of scales as $\mu \rightarrow 0, \omega(\mu)$ is given in this limit by $u_{-} /\left[2 \alpha_{1}(x) \hat{n}_{1}(x)\left(n_{-}-\hat{n}_{2}(x)\right)\right]_{\max }$.

[37] Pablo I. Hurtado, cond-mat/0507689

[38] More technically, Boltzmann equation for hard spheres (in any dimension) is unable to predict the long-time behavior of correlation functions due to the discrete character of the collision operator spectrum. In this case the long-time behavior is determined by the smallest nonzero eigenvalue of the collision operator, and this yields an exponential decay of correlations, instead of the observed power-law. 17]

[39] A.F. Andreev, A.É. Melerovich, Sov. Phys.-JETP 37, 829 (1973). 